\title{
Zero-fluoroscopy catheter ablation of focal atrial tachycardia from the noncoronary cusp
}

\author{
Bartosz Skonieczny ${ }^{1}$, Sebastian Stec², Tomasz Miszalski-Jamka \\ ${ }^{1}$ Centre for Heart Diseases, $4{ }^{\text {th }}$ Military Clinical Hospital, Wroclaw, Poland \\ ${ }^{2}$ MediNice Research and Development Centre, Rzeszow/Krosno, Poland \\ ${ }^{3}$ Tomed, Krakow, Poland
}

Adv Interv Cardiol 2021; 17, 3 (65): 334-335

DOI: https://doi.org/10.5114/aic.2021.109169

A 40-year-old nonpregnant woman with symptomatic focal atrial tachycardia (FAT) (Figure $1 \mathrm{~A}$ ) refractory to medical therapy and with no other chronic conditions was referred for catheter ablation. Cardiac magnetic resonance (CMR) showed slightly enlarged atria with no other anomalies within the heart and aortic root (AR). On 24-hour Holter monitoring over 16000 supraventricular ectopic beats, including 252 FAT episodes, were recorded.

The patient was referred for zero-fluoroscopy 3D electroanatomical mapping (3D-EAM) facilitated by the EnSite NavX system (Abbott, St. Paul, Minnesota, United States). One diagnostic 10-pole catheter (ViaCath) and one ablation electrode (AE; AlCath, 4-mm gold-tip, irrigated catheter; both Biotronik, Berlin, Germany) were advanced through the femoral vein. The decapolar catheter was advanced into the coronary sinus for reference and activation mapping. Short runs of FAT were inducible by every single extrastimulus delivered. Rapid activation mapping was performed in the right and left atria through the patent foramen ovale. The earliest activation site was found to be in the para-Hisian region and interatrial septum (IAS). Therefore, we decided to examine the AR with a retrograde femoral arterial approach. The cusp was identified as noncoronary (NCC) based solely on large atrial and small ventricular intracardiac electrograms in the AR region just posterior to the mapped His bundle potential (Figure $1 \mathrm{~B}$ ). It was presumed to be the closest site to the arrhythmia's origin despite the lack of capture of the atria and ventricle by the maximum output pacing. Single radiofrequency application (60 s, 35 Watt, $42^{\circ} \mathrm{C}$ ) was delivered, resulting in non-inducibility of FAT within the first $10 \mathrm{~s}$ of delivery. No fluoroscopy was used during the procedure (procedure times: mapping, $14 \mathrm{~min}$; ablation, $1 \mathrm{~min}$; total, $30 \mathrm{~min}$ with $15 \mathrm{~min}$ of observation). On CMR at day 7, the application site in the NCC was identified by the radiologist at least $2 \mathrm{~cm}$ away from the coronary ostia and reaching $6 \mathrm{~mm}$ deep into the IAS (Figures $1 \mathrm{C}$ and D). Within 3 months no recurrent palpitations were reported by patient and no episodes of arrhythmia were detected by several applications of Holter monitoring $(4 \times 24 \mathrm{~h})$.

The 2019 European Society of Cardiology Guidelines recommend catheter ablation as the first-line treatment for recurrent or incessant FAT [1]. FAT may originate from the NCC only in $4.1 \%$ of patients [2]. Therefore, there is no consensus on the approach to such cases. Intraprocedural AR imaging is generally recommended to guide $A E$ and assess the distance to the coronary ostia. However, in our case, AR imaging had not been performed, so AE was guided solely by mapped anatomical landmarks. Zero-fluoroscopy NCC ablation has previously been described in pregnant women only [3, 4]. Our case shows that the zero-fluoroscopy 3D-EAM approach with no intraprocedural AR imaging is feasible due to the specific location of the NCC - directly next to the para-Hisian region of the IAS. Moreover, CMR confirmed that a single application may penetrate $6 \mathrm{~mm}$ deep into nonstimulated tissue and ablate the arrhythmia focus. However, further studies evaluating the safety of such an approach in comparison to intraprocedural imaging techniques are needed.

\section{Conflict of interest}

Sebastian Stec is a stockholder of MediNice S.A. and author of multiple patents for ablation and diagnostic catheters, none of which was used in the procedure reported in this paper. 
A
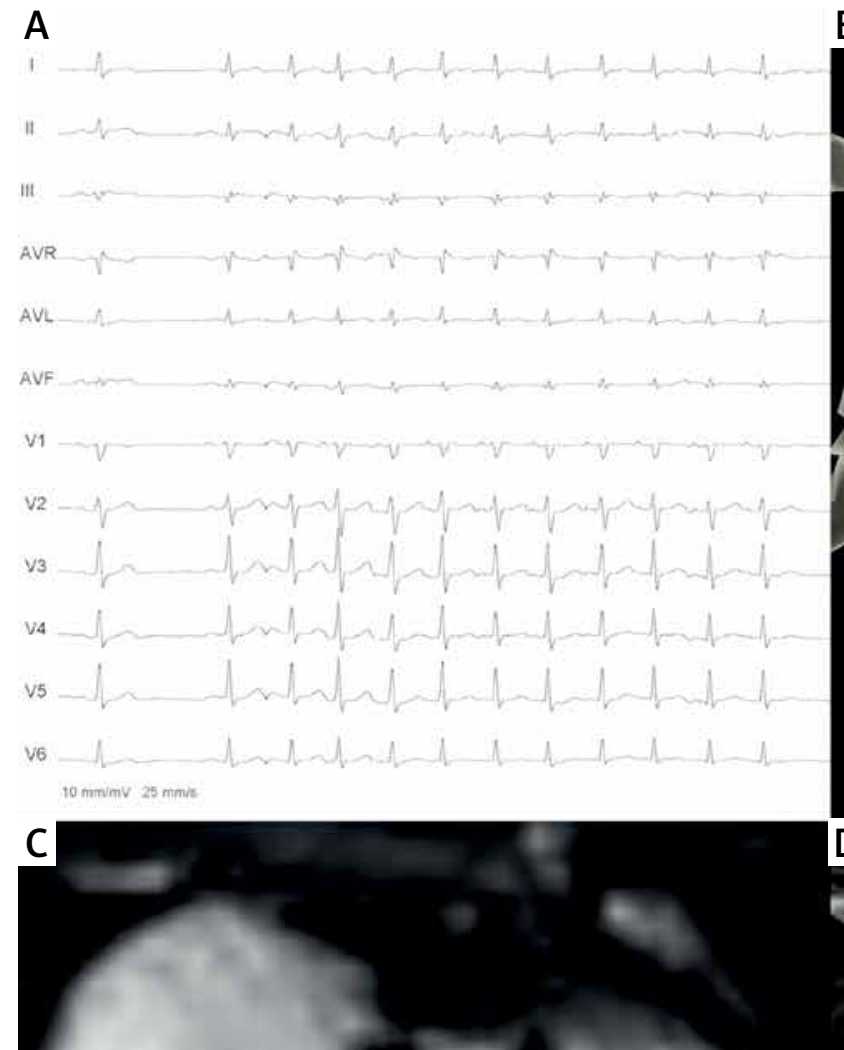

B
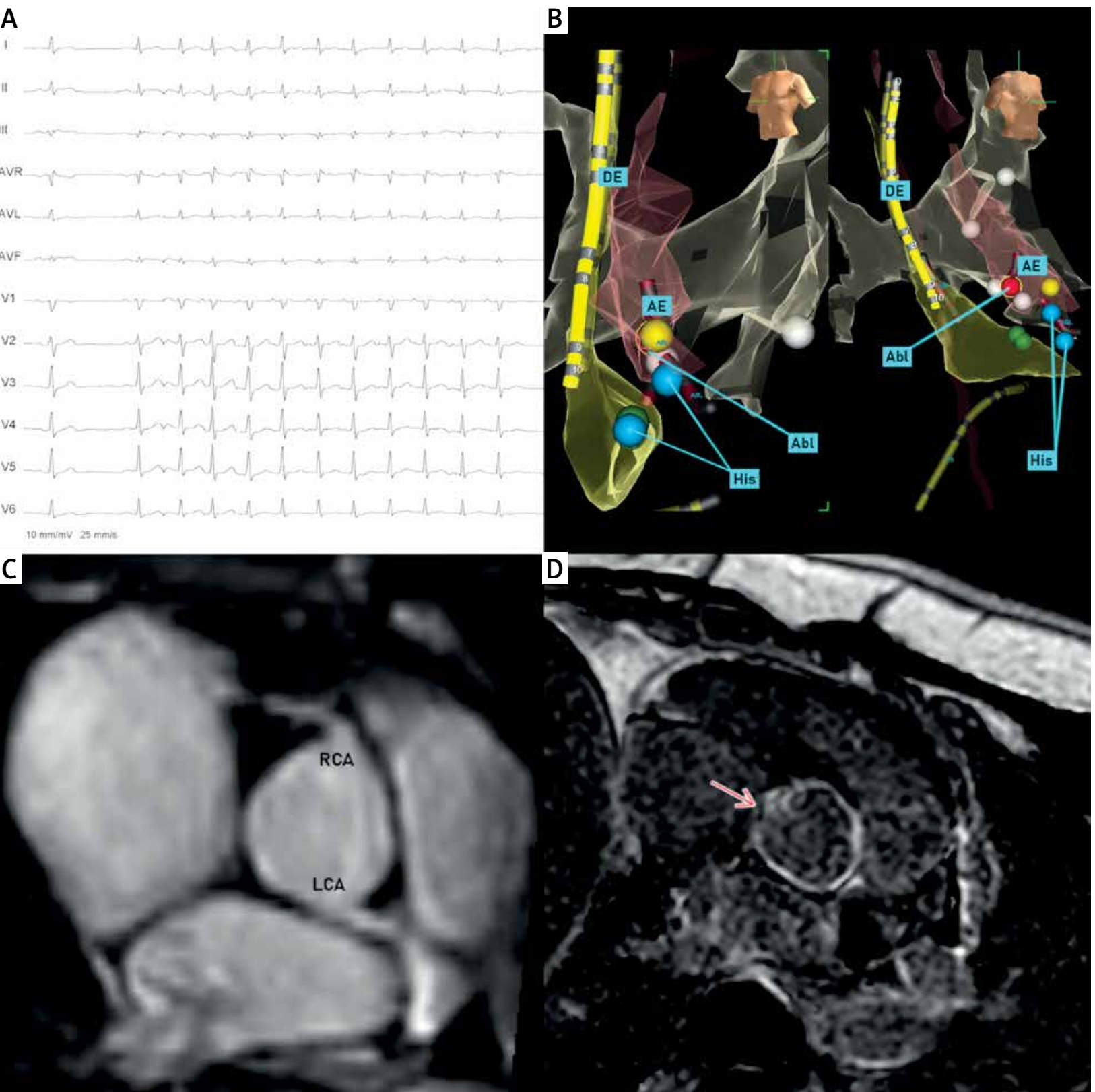

Figure 1. A - A 12-lead electrocardiogram showing normal sinus rhythm followed by the run of focal atrial tachycardia induced by a single extrastimulus. B - Three-dimensional electroanatomical mapping of the aortic root and both atria ( $30^{\circ}$ left and right anterior oblique views). The decapolar diagnostic electrode (DE) is placed in the superior vena cava. The shadow of the DE depicts the previous location in the coronary sinus. The ablation electrode $(\mathrm{AE})$ is placed in the aortic root (retrograde femoral arterial approach). Note the posterior relation of the ablation point (Abl) to far-field recording of the His bundle potential (His). C - Pre-ablation cardiac magnetic resonance showing the aortic root at the level of the coronary ostia. The left coronary artery and right coronary artery can be seen. No abnormalities are present. D - Post-ablation late gadolinium enhancement cardiac magnetic resonance. An ablation lesion (arrow) can be seen in the noncoronary cusp

\section{References}

1. Brugada J, Katritsis DG, Arbelo E, et al. 2019 ESC Guidelines for the management of patients with supraventricular tachycardia. Eur Heart J 2020; 41: 655-720.

2. Ouyang F, Ma J, Ho SY, et al. Focal atrial tachycardia originating from the non-coronary aortic sinus. Electrophysiological characteristics and catheter ablation. J Am Coll Cardiol 2006; 48: 122-31.
3. Karbarz D, Stec PJ, Deutsch K, et al. Zero-fluoroscopy catheter ablation of symptomatic pre-excitation from non-coronary cusp during pregnancy. Kardiol Pol 2017; 75: 1351.

4. Barros CBS, Chokr MO, Pisani C, et al. Catheter ablation of atrial tachycardia on the non-coronary aortic cusp during pregnancy without fluoroscopy. HeartRhythm Case Rep 2018; 4: 566-9. 